\title{
Clinical Evaluation of Fused/Ankylosed Hip with Severe Flexion Deformity after Conversion to Total Hip Arthroplasty
}

\author{
Saroj Kumar Suwal, ${ }^{1}$ Peng Songming, ${ }^{1}$ Luo Gang, ${ }^{1}$ Huang Kui ${ }^{1}$ \\ 'Department of Orthopedics, The First People Hospital of Jingzhou, Yangtze University, Jingzhou, 434000, Hubei, P. R. \\ China.
}

\section{ABSTRACT}

Introduction: Fused or Ankylosed hip is late complication of chronic inflammatory disorder with progressive changes in and around articular as well as periarticular structures with alteration in bioforce line of body which later lead to severe flexion deformity of joint. This not only results decreased movements of hip, it's also increase pain around the hip, back and contralateral hip.

Methods: Retrospectively, all patients aged 18 years or older undergoing THA between June 2006 to June 2012 were reviewed with selection criteria. The five ankylosed hips (three left and two right) with severe flexion deformities which ankylosed spontaneously were successfully converted to THA at time period of 2006 to June 2012. Range of motion, Harris Hip Score and flexion deformity angle at preoperative, postoperative and follow-up periods were used as evaluation.

Results: Mean follow up is 42 months. Mean HHS increased from $21.6 \pm 4.97$ to $81.8 \pm 4.02$ points with one excellent, two good and two fair cases. The FDA is corrected to mean $8^{\circ} \pm 10.95$ postoperatively and $4^{\circ} \pm 5.47$ at final follow up from $81.6^{\circ} \pm 4.39$ with two hips of $10^{\circ}$ residual deformity. Hip ROM is improved as flexion $70^{\circ}$ to $100^{\circ}$, adduction $10^{\circ}$ to $20^{\circ}$, abduction $10^{\circ}$ to $30^{\circ}$, internal rotation $5^{\circ}$ to $10^{\circ}$ and external rotation $2^{\circ}$ to $50^{\circ}$ from $0^{\circ}$ activity. As complications, one hip had loose prosthesis, two had early postoperative dislocations, one had Deep Vein Thrombosis and one had femoral nerve palsy with quadriceps weakness.

Conclusions: THA is an effective treatment for ankylosed hip with severe flexion deformity although complications are noted more than routine hip arthroplasties.

Keywords: ankylosed hip; fused hip; severe flexion deformity; total hip arthroplasty.

\section{INTRODUCTION}

Bony or fibrous ankylosis of hip is the spontaneous fusion with loss of both active and passive motion of the hip joint completely. The etiology of ankylosis of the hip is associated with trauma, infection, hemophilia, degenerative arthritis and ankylosing spondylitis. The ankylosis of the hip dramatically alters gait as well as the biomechanics of the adjacent joint including the change in bio-force line along lumbosacral spine, ipsilateral knee, contralateral hips and knees. ${ }^{1}$ This progressive change in and around articular and periarticular structures of hip gradually alter in function of hip especially flexion deformity as a late complication with progressive increase in intensity of pain at the hip, back and contralateral hip as well. For these reasons, the ankylosed hips were converted to

Correspondence: Dr. Huang Kui, Department of Orthopedics, The First People Hospital of Jingzhou, Yangtze University, Jingzhou 434000, Hubei P. R. China. Email: 2232813795@qq.com, Phone: +8613972334008 . 
Total Hip Arthroplasty (THA) aiming to retain functional improvement and better quality of life. ${ }^{2-4}$ In this study, we reviewed our experience and evaluate clinically of those hips.

\section{METHODS}

Retrospectively, we reviewed all patients aged 18 years or older undergoing THA between June 2006 to June 2012 after approval for the review and study by the human ethical committee according as per regulations of the First People Hospital of Jingzhou, Yangtze University. All informed consents were taken from the patient before study.

The inclusion criteria were (1) progressive disabling pain in the low back or knee, loss of function caused by immobility or malposition of hips, severe limping and walking disability. (2) All hips which were ankylosed spontaneously without any operative intervention. (3) The preoperative radiographs and CT showed fused hip with bony trabeculae. (4) The flexion deformity angle of hip is equal or greater than $60^{\circ}$. The exclusion criteria included (1) infection, (2) tumors, (3) bleeding diathesis, (4) abductor muscle dysfunction, (5) patients with less than 12-month follow-up, and (6) pregnancy.

Altogether five hips fulfilled the inclusion and exclusion criteria with three left and two right sided. Among them four fused hips were diagnosed as ankylosing spondylitis(AS) whereas one was Rheumatic Arthritis (RA) with two males and three females with age group $36 \sim 55$ years (mean 43.2 years) were included study. The mean duration of ankylosis before conversion was 12 years (range, 5-15 years). The activity was $0^{\circ}$ and FDA were $65^{\circ}-86^{\circ}$ with $76^{\circ}$ average. All of them had trouble in walking upright, excessive extension of cervical vertebrae, and varying degrees of chest and lumbar kyphosis. Clinical and radiologic evaluations were done at preoperative, postoperative and follow ups (third, sixth, and twelve months then yearly thereafter) periods where HHS and FDA were used for better clinical outcome. ${ }^{5}$

Preoperative Management: Non-Steroidal AntiInflammatory and immunosuppressant were stopped two weeks' prior surgery. Physical examination and soft tissue assessments around the hips were done thoroughly. Electromyography for muscle strength around the hip, especially gluteus medius muscles and $X-R a y$ assessment of spinal column, pelvis, femur, knee (lateral) and CT were performed to understand fusion of hip joint, malformation and acetabular location. Routine duplex ultrasonography was performed to assess the risk for Venous thromboembolism.
Operative Method: Under general anesthesia, patient was positioned in the lateral decubitus position. Improved Lateral Hip Harris Greater Trochanteric Osteotomy approach with curved the incision anteriorly by $90^{\circ}$ at the greater trochanter level was made. ${ }^{6}$ With Layer by layer incision, joint capsule was exposed. The edges of the neck of femur and surrounding soft tissues were cleared and excised. Acetabulum, ischium and lesser trochanter were exposed respectively. Subperiosteal dissection of carotid-femoral junction of pelvis done and two wide Hoffmann retractors were placed at superior and inferior of femoral neck for good exposure. Attention and protection of femoral blood vessels, nerves, and sciatic nerve were taken. About one centimeter away from pelvis, perpendicular to the axis of femoral neck, the first osteotomy was performed. From the first osteotomy line, about $5 \mathrm{~mm}$ closer to pelvis, the second femoral neck wedge osteotomy was performed antero-inferiorly to retain sufficient bone coverage behind the acetabulum. With small incision at the anterior iliac crest, the tensor fasciae latae muscle and rectus in bone were released. Acetabular reaming, medullary cavity milling, filing and sharing were done simultaneously. The femoral head and also acetabular cartilage remnants were removed thoroughly. To correct the spine and pelvis deformity, acetabular cup outreach and femoral neck angle were adjusted. A cementless acetabular cup was used in all hips (Zimmer, Warsaw, Ind). The femoral canal was reamed, and the conical femoral stem was inserted. One cemented and four cementless hip stem were used with $32 \mathrm{~mm}$ ceramic ball heads. Femoral prosthesis was used for the longer neck length and kept offset as much as possible to maintain joint stability and optimization the function of gluteus medius muscle. Intraoperative $x$-ray was used to confirm the position and angle of prosthesis while the hip joint was kept flexed approximately at $45^{\circ}$, so as to avoid stretching of the femoral nerve. Trial reduction was performed, and leg length discrepancy was assessed. After the joint reduction, trochanteric reattachment was performed with Harris Four-Wire Technique.

Post-Operative Management: Neurovascular assessment was performed postoperatively. The position of hip was kept in $45^{\circ}$ flexion with a pillow under thigh. Isometric quadriceps strengthening exercise, knee ROM and Ankle ROM were performed under regular guidance of Physiotherapy and Rehabilitation Department.

Statistical analysis: The data analysis was performed using SPSS 13.0 (SPSS Inc., Chicago, IL, USA) software. A paired t test was used to compare HHS and FDA between preoperative and postoperative period. The $\mathrm{P}$ value $<0.05$ was considered significant. 


\section{RESULTS}

Average follow-up period was 42 months, range 3846 months (Table 1). Postoperative Mean Harris Hip Score (HHS) increased from $21.6 \pm 4.97$ to $81.8 \pm 4.02$ points which was significantly improved (Table 2) with one excellent case, two good cases and two fair cases. After surgery, two patients had $20^{\circ}$ residual Flexion Deformity Angle (FDA) after which later decreased to $10^{\circ}$ with regular physiotherapy at the time of final follow-up and three patients required single cane for long-distance walking. FDA was corrected to mean $8^{\circ} \pm 10.95$ postoperatively and $4^{\circ} \pm 5.47$ at final follow up which was $81.6^{\circ} \pm 4.39$ at the time of presentation. They were statistically significant differences as compared to preoperative FDA, with postoperative and final follow-up FDA $(p<0.05)$ indicating the surgery was effective and the flexion deformity correction was significant as well.

\begin{tabular}{|cccccccc|}
\hline \multicolumn{7}{|c|}{ Table } & 1. Characteristic of Patients. \\
\hline Case & Age & Gender Side & $\begin{array}{c}\text { Diag } \\
\text { nosis }\end{array}$ & BG & $\begin{array}{c}\text { Surgery } \\
\text { Date }\end{array}$ & $\begin{array}{c}\text { FU } \\
\text { (month) }\end{array}$ \\
I & 38 & M & L & AS & Y/Y & 2008.6 & 38 \\
II & 65 & F & L & RA & Y/Y & 2010.12 & 42 \\
III & 55 & F & R & AS & Y/Y & 2011.9 & 40 \\
IV & 44 & M & R & AS & Y/N & 2009.3 & 46 \\
V & 43 & F & L & AS & Y/Y & 2010.3 & 44 \\
Mean & 43.2 & & & & & & 42 \\
\hline
\end{tabular}

(AS = ankylosing Spondylosis; RA=Rheumatic Arthritis,

$F U=$ Follow Up; BG=Bony Growth)

\begin{tabular}{|c|c|c|c|c|c|c|}
\hline \multirow{2}{*}{ Case } & \multirow{2}{*}{ Diagnosis } & \multicolumn{2}{|c|}{ HHS(points) } & \multicolumn{3}{|c|}{$\operatorname{FDA}\left({ }^{\circ}\right)$} \\
\hline & & Preoperative & Post-operative & Preoperative & Post-operative & Final FU \\
\hline 1 & AS & 27 & 86 & 75 & 0 & 0 \\
\hline II & RA & 21 & 82 & 85 & 20 & 10 \\
\hline III & AS & 14 & 76 & 86 & 20 & 10 \\
\hline IV & AS & 21 & 80 & 80 & 0 & 0 \\
\hline V & AS & 25 & 85 & 82 & 0 & 0 \\
\hline Mean & & $21.6 \pm 4.97$ & $81.8 \pm 4.02^{\#}$ & $81.6^{\circ} \pm 4.39$ & $8^{\circ} \pm 10.95^{\#}$ & $4^{\circ} \pm 5.47^{\#}$ \\
\hline
\end{tabular}

\#p value $<0.05$ versus preoperative

At final follow up, the range of flexion of all hips ranged from $70^{\circ}$ to $100^{\circ}$, the adduction of hip from $10^{\circ}$ to $20^{\circ}$ and abduction from $10^{\circ}$ to $30^{\circ}$ respectively. The final arc of rotation ranged from $5^{\circ}$ to $10^{\circ}$ of internal rotation and $2^{\circ}$ to $50^{\circ}$ of external rotation. Radiologically, one patient had loose femoral prosthesis with the evidence of radiolucent lines $2 \mathrm{~mm}$ in 2 zones seen after 2 years while rest of hips showed good bony growth at femoral stem and acetabular cup. There was Two early dislocations during extension of joint on sixth and twelveth post-operative days which were relocated in close reduction under anesthesia followed by continued skin traction for 4 weeks. Hip extension was delayed and hospital stay was increased for these hips. One patient developed deep venous thrombosis in lower limbs and managed with low molecular weight heparin where as one developed femoral nerve palsy. During two years follow up, femoral quadriceps muscle strength level was gained normal.

\section{DISCUSSION}

Ankylosed hip with severe flexion deformity causes difficult in standing upright, continuous back and hip pain with increase in intensity with time. The long term decreases in normal flexion of hip cause contractures in joint capsule, ligaments, muscles and tendons resulting the changes in periarticular structures with fibrosis and bony reconstruction around the joint and surroundings. These changes decrease in movement and impairment in overall joint function. So, THA proved as an effective management improving the joint function and mobility with relieving from pain at cervical spine, lumbar spine and lower extremities. ${ }^{7}$

Several surgical approaches have been used in the past years. ${ }^{3,8}$ Modified Harris Lateral Trochanter Osteotomy is best approach with advantages as: 1 . Simple and clear anatomical level, full femoral and acetabular exposure. 2. Avoidance of wide split of lateral iliac muscle insertions which retains good function of flexion of femur post operatively 3 . Reconstruction of the greater trochanter better adjust the abductor group stability and functionality.

Previous studies had shown that THA for ankylosed hips have higher rate of complication. Dislocation is one of the most common complications after surgery 
reported incidence of $2-4.8 \% .{ }^{9-11}$ In our study also out of five, two hips had immediate early postoperative dislocations although stability test of hip along with intraoperative $\mathrm{x}$-ray is used to determine satisfactory position and angle of the prosthesis. The periarticular soft tissue conditions could be an important factor for it .Long history of severe flexion of ankylosed hip, poor condition of muscles and soft tissues around the joints and lysis of contractured muscles during the operation could have led to poor joint stability resulting high vulnerability to dislocation. The use of larger diameter ball has obvious advantages in kinematics, increase of head diameter ratio improved range of motion and reduce the dislocation rate. ${ }^{12}$ Ceramic Ball heads and its application interface further provide better stability and excellent biocompatibility of biomaterials and reduction of joint wear rate as well.

The local and extensive adhesions, contractures, tissue scarring around the joint, limb shortening may leads to contraction and distraction of femoral and sciatic nerve causing the palsy. So, soft tissue release and maintaining proper flexion of the hip in $45^{\circ}$ at intraoperative and immediate postoperative period help to prevent the acute distraction of these femoral vessels and nerves. Moreover, the practice also proves that buckling of joints did not affect the operation of prosthetic implants. The use of cementless prostheses has long-term survival with good postoperative function with less complications and in young patient, more bone mass is preserved as well as facilitate the operation. ${ }^{13}$

\section{CONCLUSIONS}

THA is an effective treatment for severe flexion ankylosed/fused hip deformity although there are high surgical complications than routine hip arthroplasties. Adequate preoperative planning, modified surgical approach, soft tissue release, maintaining flexion of hip, use of cementless prostheses and ceramic ball heads, postoperative care with regular physiotherapy improve the clinical outcome of those hips.

\section{REFERENCES}

1. Callaghan JJ, Brand RA, Pedersen DR. Hip arthrodesis. A long-term follow-up. J Bone Joint Surg Am. 1985 Dec 1;67(9):1328-35.

2. Hamadouche M, Kerboull L, Meunier A, Courpied JP, Kerboull M. Total hip arthroplasty for the treatment of ankylosed hips. J Bone Joint Surg Am. 2001 Jul 1;83(7):992-8.

3. Kim YH, Oh SH, Kim JS, LeeSH. Total hip arthroplasty for the treatment of osseous ankylosed hips. Clinical orthopaedics and related research. 2003 Sep 1;414:136-48.

4. Joshi AB, Markovic L, Hardinge K, Murphy JC. Total hip arthroplasty in ankylosing spondylitis: an analysis of 181 hips. The Journal of arthroplasty. 2002 Jun 30;17(4):427-33.

5. Harris WH, McCarthy JC, O'Neill DA. Femoral component loosening using contemporary techniques of femoral cement fixation. J Bone Joint Surg Am. 1982 Sep 1;64(7):1063-7.

6. Harris WH, Schiller AL, Scholler JM, Freiberg RA, Scott R. Extensive localized bone resorption in the femur following total hip replacement. J Bone Joint Surg Am. 1976 Jul 1;58(5):612-8.

7. Idulhaq M, Park KS, Diwanji SR, Yoon TR, Wie JS. Total hip arthroplasty for treatment of fused hip with 90 flexion deformity. The Journal of arthroplasty. 2010 Apr 30;25(3):498-e5.
8. Sathappan SS, Strauss EJ, Ginat D, Upasani V, Di Cesare PE. Surgical challenges in complex primary total hip arthroplasty. AMERICAN JOURNAL OF ORTHOPEDICS-BELLE MEAD-. 2007 Oct;36(10):534.

9. Morsi E. Total hip arthroplasty for fused hips; planning and techniques. The Journal of arthroplasty. 2007 Sep 30;22(6):871-5.

10. Swanson MA, Huo MH. Total hip arthroplasty in the ankylosed hip. Journal of the American Academy of Orthopaedic Surgeons. 2011 Dec 1;19(12):737-45.

11. Bhan S, Eachempati KK, Malhotra R. Primary cementless total hip arthroplasty for bony ankylosis in patients with ankylosing spondylitis. The Journal of arthroplasty. 2008 Sep 30;23(6):859-66.

12. Peterson ED, Nemanich JP, Altenburg A, Cabanela ME. Hip arthroplasty after previous arthrodesis. Clinical Orthopaedics and Related Research®. 2009 Nov 1;467(11):2880-5.

13. Schäfer D, Dick W, Morscher E. Total hip arthroplasty after arthrodesis of the hip joint. Archives of orthopaedic and trauma surgery. 2000 Feb 8;120(3):176-8. 